\title{
Shale compaction and statistical physics
}

\author{
G. Korvin Eötvös Lorand Geophysical Institute, 1145 Budapest, Columhus u.17, \\ Hungary
}

Received 1983 July 5 ; in original form 1982 September 1

... investigations to date have furnished strong evidence that the behavior under pressure of the purer types of argillaceous sediments, clays and shales sensu stricto, is different from that of other sediments and while variable in detail is also in a general way uniform. The importance and universality of the phenomenon of compaction in these rocks are believed to justify considerable effort to obtain as accurate an idea of the general quantitative relations between pressure and volume as the nature of the problem will permit.

H. D. Hedberg (1936)

Summary. After a short historical outline of the development of the ideas on the compaction of clays and shales it is shown that the exponential porosity depth dependence of compacted shales expressed by Athy's law can be derived using standard methods of statistical physics. The main result of the paper states that the exponential compaction law expresses the maximumentropy equilibrium state of the pores in the rock, that is compaction is an irreversible process where clay particles tend towards a statistically defined final equilibrium. Connections with the classical theory of consolidation are pointed out.

\section{Historical and problem discussion}

Athy's law, expressing the density (or porosity) decrease as a function of depth of thick pure shale columns is one of the most unanimously accepted and widely used empirical expressions in applied geophysics. With Athy's (1930a) original notations, the compaction law states that:

$D=B+A[1-\exp (-b x)]$

where $D$ is the density to be calculated, $B$ is the density of surface clay, $A$ is the maximum density increase possible, $b$ is a constant, $x$ is depth of burial. In recent usage, the compaction law is generally quoted as:

$\rho(z)=\rho_{0}+\left(\rho_{\infty}-\rho_{0}\right)[1-\exp (-k z)]$ 
with $\rho_{0}$ surface density, $\rho_{\infty}$ the upper limit of shale density, $z$ depth and $k$ is a constant. An equivalent form of this law describes the porosity decrease with depth:

$\Phi(z)=\Phi_{0} \exp (-k z)$

where $\Phi(z)$ is the porosity at depth $z, \Phi_{0}$ is the porosity at the surface. (The equivalence of these different formulations of Athy's law will be shown in Section 2.)

Most textbooks on gravity prospecting (Grant \& West 1965; Militzer \& Lindner 1976) suggest the use of Athy's rule for gravity computations. Case histories proving the applicability of this rule are reported by Ceccherini \& Finetti (1968) and Cordell (1973). Recently, the compaction rule has found other kinds of application in the geoacoustic modelling of the seafloor (Hamilton 1980; Matthews 1980), in Magara's (1976) water expulsion studies and has become a basic tool of the backstripping technique (Watts 1981) used in the reconstruction of the subsidence history of sedimentary basins.

An excellent summary of the historical development of the early ideas on the compaction of clays and shales is given by Hedberg (1936). Apparently, the first quantitative relationship connecting porosity and pressure was due to Terzaghi (1925):

$P=a G^{b}(P=$ porosity, $G=$ pressure $)$

while Hedberg $(1926,1936)$ could fit a law of the form

$P=40.22(0.9998)^{G}$

( $G$ is the pressure measured in pounds per square inch) to his Venezuelan undisturbed tertiary shale data. A discussion in 1927 between W. W. Rubey and H. D. Hedberg brought out many additional points of interest. Rubey proposed a hyperbolic type of law to describe Hedberg's Ransom well data:

$(D+B) \frac{P}{100-P}=C$

(where $D$ is the observed depth of burial, $B$ the eroded depth, $P$ the observed percentage porosity and $C$ a constant which varies in different areas) and drew a parallel between this formula and the Van der Waal's equation relating volume to pressure in an ideal gas. Both Hedberg and Athy refuted Rubey's argument, Athy (1930a) attributed the success of Rubey's hyperbolic fitting to the very few number of data.

Since the late 1930s the exponential compaction rule has almost unanimously been accepted and confirmed by ample evidence (Hedberg 1926, 1936; Athy 1930a; Dickinson 1953; Rische 1957; Nafe \& Drake 1957; Maxwell 1964; Nagumo 1965; Ozerskaya 1965; McCulloch 1965; Howell, Heintz \& Barry 1966; Kopf 1967; Ceccherini \& Finetti 1968; Avchyan \& Ozerskaya 1968; Baldwin 1971; Ozerskaya \& Semonova 1973; Cordell 1973; Anstey 1977; Hamilton 1980; Watts 1981; etc.). Characteristic orders of magnitudes for the decay constant in equations (2) and (3) are $1.46 \mathrm{~km}^{-1}$ (Athy 1930a, for Palaeozoic rocks in Oklahoma), $0.64 \mathrm{~km}^{-1}$ (Hedberg 1936, for an undisturbed tertiary shale column in Venezuela) and $0.28 \mathrm{~km}^{-1}$ (Howell et al. 1966, for an 'area of salt dome occurrence' of unspecified location). Laboratory investigations on the porosity-pressure relation (Maxwell \& Verrall 1954) are also in agreement with the exponential compaction law, for nonsedimentary rocks, however, Volarovich et al.'s (1970) high pressure studies resulted in a

$\rho=\frac{\rho_{0}}{1-\Delta V / V_{0}}$ 
law, $\Delta V / V_{0}$ being the relative change of pressure, which is more similar to Rubey's equation (6). There are some recent case histories where Athy's compaction law has apparently failed. Sz. Kilényi (1968), on the basis of 1185 density data and 224 porosity data, concluded that the density depth curves should be fitted by straight lines rather than exponentially, only one area from among the six sites studied could be fitted by Athy's equation, and even in this case, unrealistic $\rho_{0}$ and $\rho_{\infty}$ values had to be used. Vikhirov (1975) also reports a straightline law, Gladkikh \& Urazaev (1972) approximate the $\rho(z)$ and $\Phi(z)$ dependence by secondand third-degree parabolas, with correlation coefficients between 0.7 and 0.96 . Dabrowski (1976) investigates the depth dependence of the mean densities of pre-Devonian sedimentary rocks in Poland via regression lines of the forms $\rho(z)=a z+b ; \rho(z)=a z^{2}+b z+c ; \rho(z)=$ $a \log z+b$ and reports a best fit for the second-degree parabolic approximation.

Very likely, these discrepancies can be explained away by the fact that - as shown later - the Athy compaction law describes the final equilibrium state of shales after part of the water content had been expelled vertically upwards. In the case of sand --shale interbedding a significant horizontal migration of the water can take place (Magara 1976) so that the exponential compaction law should not strictly apply.

The aim of this paper is to provide a mathematically physically exact proof for the hitherto only empirically established exponential compaction law, using standard methods of statistical physics. As far as I know the only attempt to date to prove this fundamental rule was made by Nagumo (1965) who used elasticity theory and had to make a rather ad hoc assumption that the difference between pore compressibility and framework compressibility is kept constant during the process of compaction.

My motivations for using the statistical approach will become clear in Section 3. At this point, I will only mention some opinions and facts from the published literature that were of decisive importance to this study:

(1) In the discussion in 1927 between Rubey (1927a, b) and Hedberg (1927), Rubey proposed a thermodynamical analogy (the Van der Waal's equation) to shale compaction.

(2) Magara (1976) mentions that Athy's compaction law expresses a 'compaction equilibrium', the exact meaning of this equilibrium (isostatic? statistic?) is left unspecified.

(3) According to Cordell (1973): 'the density-depth relationship in thick sedimentary sequences does not lend itself to a deterministic mathematical formulation because the effects of stratigraphic layering, facies, cementation, diagenesis, and tectonic history are involved, in addition to simple compaction due to geostatic pressure. ... Nevertheless, in spite of the many variables, actual measurements of sedimentary rock density in deep drill holes indicate that density and depth sometimes can be related according to a simple mathematical law' (my italics).

(4) The system-theoretical approach - outlined by L. Kapolyi in his inaugural speech at the Hungarian Academy of Sciences (1980) - treats the processes of complicated geologic media on the principle that the evolution of closed systems always proceeds towards the maximal entropy. What $I$ do in this paper is an application of this general principle to the possibly simplest geologic process of shale compaction.

(5) The methods to be applied rely heavily upon the ideas of Litwiniszyn (1974) who described the mechanics of granular media on the principle that the 'downward migration of particles corresponds with an oppositely directed migration of empty spaces', and applied stochastic partial differential equations to express the random migration of voids.

(6) Finally, I tried to explain the interesting fact (reported by Nafe \& Drake 1957 and Sz. Kilényi 1968) that the gradient of the $\rho(z)$ line is much steeper in the shallower parts of a basin than in the deeper parts. (Similar conclusions are reported on the gradient of the seismic interval velocities, see Zsellêr 1980.) 


\section{Equivalent forms of the compaction law}

The usual form of Athy's compaction law is

$\rho(z)=\rho_{0}+\left(\rho_{\infty}-\rho_{0}\right)[1-\exp (k z)]$

where $\rho_{0}$ is the sediment density at the surface, $\rho_{\infty}$ the upper limit of shale density, $z$ the depth and $k$ a constant depending on the area.

Equation (8) can also be written as

$\rho(z)=\rho_{0} \exp (-k z)+\rho_{\infty}[1-\exp (-k z i]$.

Denote by $\Phi(z)$ the porosity at depth $z$. It is a physically reasonable fact, supported by a number of measurements, that the bulk density of porous rocks is given by the mixture rule

$\rho=\Phi \rho_{1}+(1-\Phi) \rho_{2}$

where $\rho_{1}$ is the density of the fluids filling the pores, $\rho_{2}$ is the matrix density (Nafe \& Drake 1957; Vikhirov 1965, for more than 9000 samples: Hamilton 1970; Vikhirov \& Taraskova 1975; Anstey 1977). It is also known (e.g. Militzer \& Lindner 1976) that the density $\rho_{\infty}$ figuring in Athy's law is $\rho_{\infty} \approx 2.7 \times 10^{3} \mathrm{~kg} \mathrm{~m}^{-3}$, i.e. about the same value as the matrix density $\rho_{2}$ of equation (10) (cf. Nafe \& Drake 1957).

We proceed to prove that the Athy compaction law (equation 9) with $\rho_{\infty}=\rho_{2}$ is equivalent to the porosity depth rule

$\Phi(z)=\Phi_{0} \exp (-k z)$

where $\Phi_{0}$ is the porosity at the surface.

Proof: Assume first that (11) holds. Applying the mixture rule (10) we have, after some algebra,

$$
\begin{aligned}
\rho(z) & =\Phi(z) \rho_{1}+[1-\Phi(z)] \rho_{2}=\Phi_{0} \exp (\cdots k z) \rho_{1}+\left[1 \cdots \Phi_{0} \exp (-k z)\right] \rho_{2} \\
& =\left[\Phi_{0} \rho_{1}+\left(1-\Phi_{0}\right) \rho_{2}\right] \exp (-k z)+\rho_{2}[1 \cdots \exp (-k z)] \\
& =\rho_{0} \exp (-k z)+\rho_{2}[1-\exp (-k z)]
\end{aligned}
$$

that is Athy's law (9) holds with $\rho_{\infty}=\rho_{2}$. Conversely, by expressing $\rho(z)$ in terms of $(9)$ and by the mixture rule, respectively:

$\rho_{1} \Phi(z)+\rho_{2}[1-\Phi(z)]=\rho_{0} \exp (-k z)+\rho_{\infty}[1-\exp (-k z)]$

that is, since evidently $\rho_{1} \neq \rho_{2}$,

$\Phi(z)=\frac{\left(\rho_{0}-\rho_{\infty}\right) \exp (-k z)}{\rho_{1}-\rho_{2}}+\frac{\rho_{\infty}-\rho_{2}}{\rho_{1} \cdots \rho_{2}}$.

By the assumption $\rho_{\infty}=\rho_{2}$, i.e. the constant term of (12) drops out and, by a repeated application of the mixture rule (10),

$$
\Phi(z)=\frac{\left[\Phi_{0} \rho_{1}+\left(1-\Phi_{0}\right) \rho_{2}-\rho_{2}\right] \exp (-k z)}{\rho_{1}-\rho_{2}}=\Phi_{0} \exp (-k z)
$$

and the proof has been accomplished. 
Because of the equivalence of expressions (8) and (11), it will suffice in what follows to prove Athy's law in the form

$\Phi(z)=\Phi_{0} \exp (-k z)$.

Assuming pores of identical volume, the porosity of a rock is proportional to the number of pores in a unit volume of the rock, i.e. to the density of the distribution of pores. In these terms, Athy's rule states that the pores in compacted shaly sediments are distributed in such a manner that their number in a unit volume of rock exponentially decreases with depth.

Before proving Athy's law it will be instructive to see some analogies to (11), from different branches of physics.

\section{Analogies to Athy's law}

Once we have recognized that (11) expresses the exponential decrease with depth of the population density of pores in a compacted shale column, it is easy to recall some analogies to this rule from different branches of physics. The most evident analogy is the barometric altitude equation due to Boltzmann, expressing the density $n(z)$ of the air at an altitude $z$ as

$n(z)=n(0) \cdot \exp \left[-\frac{m g z}{k T}\right]$

where $m$ is the mass of a single gas molecule, $g$ the gravity acceleration, $k$ the Boltzmann constant and $T$ the absolute temperature.

A very similar formula describes the density of small colloidal particles in a suspension:

$n(z)=n(0) \cdot \exp \left[-\frac{\left(\rho-\rho_{0}\right) v g z}{k T}\right]$

Here, $v$ is the volume of a small particle, $\rho$ and $\rho_{0}, \rho>\rho_{0}$, are the densities of the particles and of the fluid, respectively. The thermodynamical derivation of these formulae can be found in standard textbooks on statistical physics (see, e.g. Macke 1962 and Sommerfeld 1962 for equations 13; Károlyházi, Marx \& Nagy 1965 for equation 14). The proof is generally based on the Boltzmann statistics, i.e. on the assumption that the gas particles move independently of each other and the whole system tends toward its most probable (maximum entropy) state. An interesting derivation of the barometric altitude equation on the basis of information theory was given by Fritz (1967).

There also exists an elementary proof to (13), in terms of a simple separable differential equation (Kneschke 1960), in course of the proof, however, we have to borrow the BoyleMariotte law from gas dynamics, that is basically this proof as well is of a statistical nature.

A surprisingly close analogy to the shale compaction law has been found by Medwin (1970) who used a portable pulse-echo, pulse-scatter acoustical system to measure the numbers of bubbles of radius approximately $180-18 \mu \mathrm{m}$ at sea. The study was made at various depths to $50 \mathrm{ft}$ in isothermal coastal waters and it was found that the number of bubbles of radius less than $60 \mu \mathrm{m}$ follows an exponential depth dependence exp $(-z / L)$ with $L$ approximately $7 \mathrm{~m}$. While Medwin (1970) did not provide an explanation for this interesting finding, the result can very likely be explained by following up the statistical approach of Floyd (1981).

We realize that the above-mentioned analogies between shale-compaction and other physical phenomena would seem for most readers rather strange and far-fetched. Indeed, apparently there is very little in common between the clay (or fluid) particles in a consoli- 
dated rock and the microbubbles in coastal ocean waters. Recall, however, that Terzaghi himself (1965) called attention to the analogy between his 1-D consolidation equation (49) and the equation of heat conduction; Terzaghi \& Fröhlich (1936) present further analogies from different branches of physics (diffusion of substances dissolved in liquids, diffusion of gases, propagation of electric current in cables, and movement of solid bodies through a stationary viscous liquid).

Analogies, of course, usually do not have more than a heuristic value. In the proof of Athy's compaction law in the next section we shall only use the well-established methods of statistical physics without any further reference to the analogies given in the present section.

\section{Derivation of the shale compaction law on the basis of statistical physics}

We start out from the assumption that the decrease in porosity of shales during compaction is largely due to the expulsion of free and adsorbed water with some mechanical rearrangement of particles towards a denser system of packing (Hedberg 1936; Magara 1976). The following simple depositional model is used (Anstey 1977), for fine-grained sediments. Products of land erosion are brought down to the sea by rivers and are deposited. At the time of deposition, the water content by volume of a clay may be 60 per cent or even more. Since the density of the mix is larger than that of the seawater it replaces, but less than a pure clay density, there is a compacting effect acting on the clay, squeezing out the water back into the sea. As more clay is deposited on top, more compaction occurs below, and more water is expelled. Finally, during geologic time, the fine-grained muds become clay rocks and shales under the influence of compaction.

If we focus our attention to a microscopic plate-like particle of the originally loose collection, the weight of the overburden bends this small particle so that its previously free end is now touching another particle. At the point of contact, attractive forces bond the two particles together so that even when the applied force is removed, the particles remain bonded because the bonding forces are strong enough to hold them in their bent configuration (cf. Weyl \& Ormsby 1960; Walker 1982). This microscopic description of the compaction behaviour of a single plate-like particle is an equivalent way of saying that a small pore (or void, or fluid) particle' has just moved one step upwards (as in the model of Litwiniszyn 1974). In other words, the compaction of shale can be described in terms of the upward migration of pores.

Consider a rectangular prism of unit cross-section of the compacted shale down to the basement at depth $Z_{0}$, and suppose in its present state its mean porosity is $\bar{\Phi}$, i.e. it contains a fractional volume $\bar{\Phi} Z_{0}$ of fluid and a volume $(1-\bar{\Phi}) Z_{0}$ of solid clay particles.

We will neglect the water completely squeezed out of the rocks, and assume the evolution of the system is ergodic, i.e. it tends towards the same maximum-entropy final state no matter what the initial conditions had been. (The compaction problem cannot be solved without this assumption, since there exists no deterministic description of the sedimentation process. Sedimentation is usually considered as a random succession of deposition and erosion episodes, see Tipper 1983.) Assume that at time $t=0$ the initial condition was that shown in Fig. 1, i.e. a prism of water of unit cross-section, height $\bar{\Phi} Z_{0}$ and density $\rho_{1}$ is overlain by a prism of solid clay particles of height $(1-\bar{\Phi}) Z_{0}$ and density $\rho_{2}, \rho_{1}<\rho_{2}$. Suppose the prism of water can be divided into $\mathfrak{N}$ particles, each of volume $\Delta V$ and these particles begin to migrate upwards independently of each other, until the equilibrium (maximum entropy) state is reached.

The initial potential energy of the system is

$E=\mathfrak{\Re} \cdot \Delta V \cdot g\left(\rho_{2}-\rho_{1}\right)(1-\bar{\Phi}) Z_{0}$. 


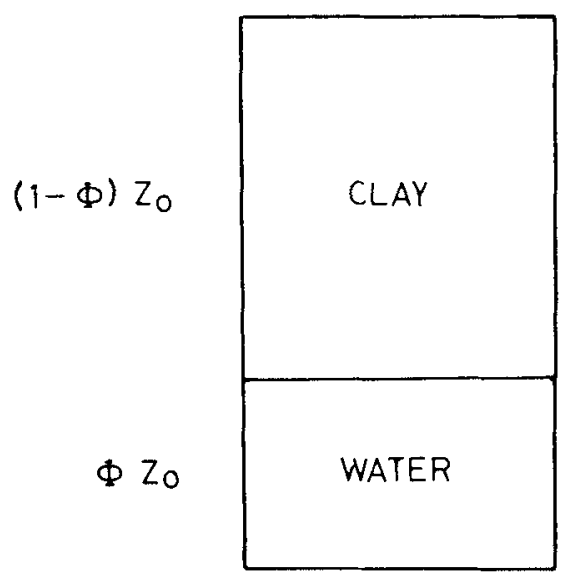

Figure 1. The initial stage of deposition.

Also, the initial porosity and particle number are connected by

$$
\mathfrak{N}=\frac{Z_{0} \bar{\Phi}}{\Delta V} .
$$

The proof of the compaction law will be carried out in several steps.

(1) We introduce a coordinate system $(x, y, z), z$ pointing downwards, and divide the prism $\mathrm{H}$ of Fig. 2 into $N$ equal thin slabs of thickness $\Delta z=Z_{0} / N$. Denote the $i$ th slab by $\gamma_{i}(i=0,1, \ldots, N-1)$, i.e.

$\gamma_{i}=\| P(x, y, z) \mid x \in[0,1] ; y \in[0,1] ; i \Delta z \leqslant z<(i+1) \Delta z \rrbracket i=0,1, \ldots, N-2$

$\gamma_{N-1}=\llbracket P(x, y, z) \mid x \in[0,1] ; y \in[0,1] ;(N-1) \Delta z \leqslant z \leqslant Z_{0} \rrbracket$

where $P(x, y, z)$ are the points of the prism $\mathrm{H}$.

(2) We divide the entire prism $\mathrm{H}$ into $N^{*}=Z_{0} / \Delta V$ small cubes, we assume the pores can only be in some of these positions (states). We also assume that $\mathfrak{N} \ll N^{*}$ which is always satisfied if $\bar{\Phi}$ is sufficiently small (cf. 16).

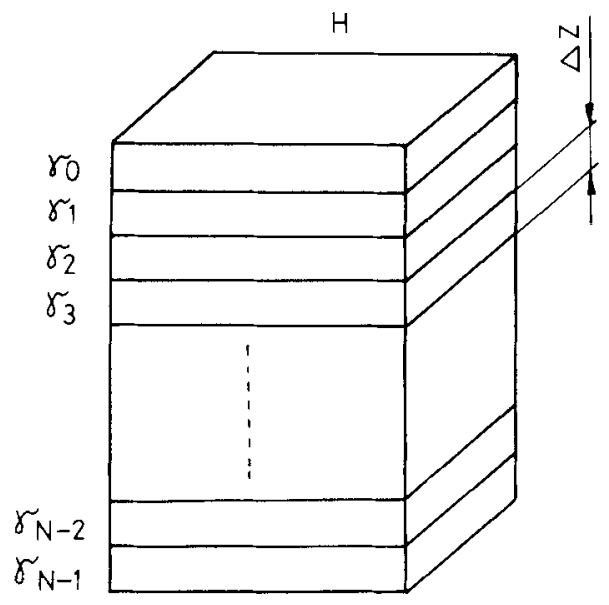

Figure 2. Definition of the macroscopic states. 
(3) We rank the $N^{*}$ possible states into $N$ groups: a pore is said to belong to the group of states $\gamma_{i}$ if and only if its centre $P(x, y, z)$ lies within the slab $\gamma_{i}$. This implies the group $\gamma_{i}$ contains altogether $G=\Delta z / \Delta V$ states.

(4) Suppose that in some permitted state of the rock exactly $N_{i}$ pores can be found in the group $\gamma_{i}$. The numbers $N_{i}$ should fulfil the following constraints, expressing the conservation of pore-particle number, and the conservation of total potential energy:

$\sum_{i=0}^{N-1} N_{i}=\mathfrak{N}$

$\sum_{i=0}^{N-1} \epsilon_{i} N_{i}=E$

where $E$ is the total energy ( $c f .15) ; \epsilon_{i}$ is the potential energy of a single pore particle in group $\gamma_{i}$, to be defined later.

(5) The set of numbers $N_{i}$ completely determine the macroscopic state of the distribution of pores inside the prism $\mathrm{H}$. We determine next the thermodynamical probability $\Gamma$ of the given macroscopic state $\left\{N_{i}\right\}$, i.e. we enumerate how many microscopic realizations of this state exist. (For terminology and method see standard textbooks on statistical physics, e.g. Sommerfeld 1962; Landau \& Lifshitz 1981.) If all the groups $\gamma_{i}$ are treated as independent and $\Gamma_{i}$ denotes the thermodynamic probability of the $i$ th group then

$\Gamma=\prod_{i=0}^{N-1} \Gamma_{i}$.

(6) We assume the number of pores $N_{i}$ actually being in group $\gamma_{i}$ is much less than the number of possible positions $G$ within a group $\left(N_{i}^{2} \ll G\right)$, in themselves, however, the $N_{i}$ s are sufficiently large.

(7) There are $\left(\begin{array}{l}G \\ N_{i}\end{array}\right)$ possible ways of distributing the $N_{i}$ pores among the $G$ states of the group $\gamma_{i}$, for we do not distinguish between pore particles.

So, the thermodynamic probability that the group $\gamma_{i}$ would contain $N_{i}$ pore particles is given by

$\Gamma_{i}=\left(\begin{array}{c}G \\ N_{i}\end{array}\right)=\frac{G !}{N_{i} !\left(G-N_{i}\right) !}$.

(8) In statistical physics the entropy of the distribution $\left\{N_{i}\right\}$ is defined, apart from a constant factor, as the logarithm of the thermodynamic probability:

$S=\log \Gamma=\sum_{i=0}^{N-1} \log \Gamma_{i}$.

Since the numbers $G, N_{i}, G-N_{i}$ are large, (22) can be approximated by Stirling's formula:

$n ! \approx\left(\frac{n}{e}\right)^{n} \sqrt{2 \pi n}(n \gg 1)$

that is, recalling that $(1+x / n)^{n} \rightarrow \exp (x)$ if $n \rightarrow \infty$, and keeping only the dominating terms, the thermodynamic entropy becomes, apart from a constant multiplication factor and an 
additive term

$S=\sum_{i=0}^{N-1} N_{i} \log \frac{e G}{N_{i}}$

It should be noted that the entropy given by (25) is intimately connected with Shannon's entropy of information theory. Indeed, if $p_{i}(i=0,1, \ldots, N-1)$ denotes the probability that a pore particle belongs to group $\gamma_{i}$, and $\mathfrak{N}$ and the numbers $N_{i}$ are large, we have $N_{i} \approx \mathfrak{N} \cdot p_{i}$ and it is easy to check that (25) becomes, in terms of the probabilities

$S=$ constant $-\mathfrak{M} \cdot \Sigma p_{\boldsymbol{i}} \log p_{\boldsymbol{i}}$

(9) Let us denote the average number of pore particles in group $\gamma_{i}$ by $\vec{n}_{i}$, that is

$\bar{n}_{i}=\frac{N_{i}}{G}$.

Substituting (26) into (25)

$S=G \cdot \sum_{i=0}^{N-1} \bar{n}_{i} \log \frac{e}{\bar{n}_{i}}$

while the pore number and energy constraints (19), (20) become

G. $\sum_{i=0}^{N-1} \bar{n}_{i}=\mathfrak{M}$

G. $\sum_{i=0}^{N-1} \bar{n}_{i} \epsilon_{i}=E$.

(10) As usually done in Boltzmann statistics, we assume that after a sufficiently long time the pore particles will be found in that equilibrium (or final) state where the entropy (27) is maximal (see Kapolyi 1980; Landau \& Lifshitz 1981). To determine this state we have to maximize the entropy (27) subject to the constraints (28), (29).

Since the functions $S$ and $a S+b$, where $a>0$ and $b$ are arbitrary constants, attain their maxima for the same point of the $(N-2)$-dimensional domain determined by (28) and (29), the neglection of the constant factors in the derivation of $S$ is justified. Also, by (25a), the optimization of the thermodynamic entropy $S$ or of the Shannon entropy would lead to the same result.

(11) Introducing the Lagrange multipliers $\alpha, \beta$ the following derivatives should vanish:

$\frac{\partial}{\partial \tilde{n}_{i}}\left(S+\alpha \frac{\mathfrak{N}}{G}+\beta \frac{E}{G}\right)=0(i=0,1, \ldots, N-1)$

that is

$G\left(-\log \bar{n}_{i}+\alpha+\beta \epsilon_{i}\right)=0(i=0,1, \ldots, N-1)$

and

$\bar{n}_{i}=\exp \left(\alpha+\beta \epsilon_{i}\right)$

where $\alpha, \beta$ are unknown constants.

(12) The constants $\alpha, \beta$ are determined from conditions (28), (29). First, observe that by 
the definitions (17), (18) of the group $\gamma_{i}$ the potential energy, due to buoyancy, of a single pore particle in group $\gamma_{i}$ is given, for $\Delta z \ll 1$, by

$\epsilon_{i}=g\left(\rho_{2}-\rho_{1}\right) \Delta V \cdot i \cdot \Delta z$.

Substituting expression (32) into (28) and (29) and recalling that $G=\Delta z / \Delta V$, we obtain

$\Delta z \cdot \sum_{i=0}^{N-1} \bar{n}_{i}=\exp \alpha \cdot \sum_{i=0}^{N-1} \exp \left[\beta g\left(\rho_{2}-\rho_{1}\right) \Delta V \cdot i \cdot \Delta z\right] \cdot \Delta z=\mathbf{n} \cdot \Delta V$

$\Delta z \cdot \sum_{i=0}^{N-1} \bar{n}_{i} \epsilon_{i}=\exp \alpha \cdot \sum_{i=0}^{N-1} \exp \left[\beta g\left(\rho_{2}-\rho_{1}\right) \Delta V \cdot i \cdot \Delta z\right]$

$$
\times\left[g\left(\rho_{2}-\rho_{1}\right) \Delta V \cdot i \cdot \Delta z\right] \cdot \Delta z=E \cdot \Delta V .
$$

If we let $N \rightarrow \infty, \Delta z \rightarrow 0$ and the finite sums in (34) and (35) become integrals

$\exp \alpha \cdot \int_{0}^{Z_{0}} \exp \left[\beta g\left(\rho_{2}-\rho_{1}\right) \Delta V \cdot z\right] d z=\mathfrak{N} \cdot \Delta V$.

$\exp \alpha \cdot \int_{0}^{Z_{0}} \exp \left[\beta g\left(\rho_{2}-\rho_{1}\right) \Delta V \cdot z\right] g\left(\rho_{2}-\rho_{1}\right) \Delta V \cdot z \cdot d z=E \cdot \Delta V$

(13) By inspecting the form (32) of the number $\vec{n}_{i}$ it is evident that $\beta$ cannot be positive since this would result in a porosity increasing with depth that certainly cannot be a stable configuration in the gravitational field of the Earth. So, we can restrict ourselves to those solutions to (36) and (37) where $\beta \leqslant 0$. It is easy to check that for $\beta=0$ we get contradictory equations for exp $\alpha$ from the system (36) and (37) unless $\bar{\Phi}=0.5$.

Assuming, that

$\bar{\Phi}<0.5$

we only have to deal with those solutions where $\beta<0$.

(14) Introduce the further notations

$a=g\left(\rho_{2}-\rho_{1}\right) \Delta V$.

$\bar{E}=\frac{E}{\mathfrak{N}}$

where $\bar{E}$ is the average potential energy of a single pore. Since $\beta<0$, the upper limit of the integrals in (36) and (37) can be taken as $+\infty$ if $Z_{0}$ is sufficiently large and the system (36) and (37) simplifies to

$$
\begin{aligned}
& -\exp \alpha \cdot \frac{1}{a \beta}=\mathfrak{N} \Delta V . \\
& \exp \alpha \cdot \frac{1}{a \beta^{2}}=E \Delta V .
\end{aligned}
$$

that is, by means of (39), (40) and (16)

$$
\exp \alpha=\frac{\bar{\Phi}}{1-\bar{\Phi}}
$$


$\beta=-\frac{1}{\vec{E}}$

and, by (32)

$\bar{n}_{z}=\frac{\bar{\Phi}}{1-\bar{\Phi}} \exp \left[-\frac{z}{(1-\bar{\Phi}) Z_{0}}\right]$.

(15) Recalling the definition of $G$ and that of the numbers $\bar{n}_{i}(26)$, the expected number of pores inside a slab $(z, z+d z)$ of the prism $\mathrm{H}$ is

$N_{z}=\frac{d z}{\Delta V} \bar{n}_{z}$

their total volume is $N_{z} \cdot \Delta V=d z \cdot \bar{n}_{z}$, that is the porosity of this slab is $\Phi_{z}=N_{z} \Delta V / d z$ implying that the porosity-depth dependence of compacted shale is expressed by the simple rule

$\Phi(z)=\frac{\bar{\Phi}}{1-\bar{\Phi}} \exp \left[-\frac{z}{(1-\bar{\Phi}) Z_{0}}\right]$.

Identifying the first factor $\bar{\Phi} /(1-\bar{\Phi})$ in (46) with the surface porosity $\Phi_{0}$ of shales:

$\Phi_{0}=\frac{\bar{\Phi}}{1-\bar{\Phi}}$

and solving for $\bar{\Phi},(46)$ can be rewritten in terms of surface porosity as

$\Phi(z)=\Phi_{0} \exp \left[-\frac{\left(1+\Phi_{0}\right) z}{Z_{0}}\right]$

an equation that reproduces Athy's compaction law and qualitatively expresses the inverse dependence of compaction rate on basin depth, reported by Nafe \& Drake (1957) and Sz. Kilényi (1968). Of course, the surface porosity $\Phi_{0}$ and average porosity $\bar{\Phi}$ figuring in (46) and (48) are both functions of the total amount of water squeezed out of the shale, that is of geologic time, temperature, granulometry and possibly of depth as well. However, if the surface porosity $\Phi_{0}$ of the shale is known and is fairly constant, the inverse relation of compaction rate to basin depth seems to be an exact law.

\section{Connections with the classical theories of consolidation}

It should be noted that the principle of maximum entropy only determines the final, asymptotic state of the systems studied as the time $t$ tends to infinity, it does not, however, provide the time-history of the evolution towards this final state (see the remarks on the ergodic theorems in Reed \& Simon 1972). Obviously, any large deviation from the maximal entropy state (i.e. from Athy's law) reflects some discrepancy (interbeddings, recent tectonic movements), or it simply refers to the fact that the shale column is too young and the equilibrium state has not been reached as yet. In the latter case one would even expect some quantitative relationship between the deviation of the porosity-depth curve from Athy's law and the age of the shale. 
In studies of this kind the time-course of the compaction can be described by the 1-D consolidation equation of Terzaghi (1923). Using the notations and terminology of Terzaghi (1965), this equation states that the excess hydrostatic pressure $u(z, t)$ at time $t$ and depth $z$ satisfies the partial differential equation

$\frac{\partial u(z, t)}{\partial t}=\frac{k}{\gamma_{\mathrm{w}} \cdot m_{\mathrm{vc}}} \cdot \frac{\partial^{2} u(z, t)}{\partial z^{2}}$

subject to appropriate boundary conditions. In (49) $k$ is the coefficient of permeability, $\gamma_{w}$ the density of the water, $m_{\mathrm{vc}}$ is the coefficient of volume decrease. Equation $(49)$ can be derived from Darcy's law if the permeability $k$ is taken as a constant, independent of depth, pressure or porosity. According to Terzaghi, the porosity $\Phi(z, t)$ is connected to the excess hydrostatic pressure as

$\frac{\partial \Phi}{\partial t}=m_{\mathrm{vc}} \cdot \frac{\partial u}{\partial t}$.

The main obstacle to the application of the consolidation equation (49) for shale-compaction studies seems to be that in the case of larger variations of the porosity with depth the constancy of $k$ in the derivation of (49) from Darcy's law cannot be justified. Indeed, as is well known from hydrology and reservoir mechanics, in sedimentary rocks the permeability is always interrelated with porosity, even though the exact nature of this interrelatedness is poorly understood and is generally expressed through further empirical constants. For example, in the classical Kozeny-Carman equations (Scheidegger 1963) the $k$-- $\Phi$ dependence is expressed by means of the specific internal surface area; in Wyllie \& Rose (1950) by means of the hydraulic tortuosity and the effective channel cross-section; in Schopper's (1966) random network model $k$ and $\Phi$ are connected via a set of parameters, such as tortuosity, expected channel cross-section, expected hydrodynamic shape factor, network constant, etc. If $k$ does depend on $z$ (through its porosity-dependence) the consolidation equation (49) should be written as

$\frac{\partial u}{\partial t}=\frac{1}{m_{\mathrm{vc}} \gamma_{\mathrm{w}}} \cdot \frac{\partial}{\partial z}\left(k \frac{\partial u}{\partial z}\right)$.

In order to use (51) to predict the spatial-temporal behaviour of the porosity during the compaction process the excess hydrostatic pressure $u(z, t)$ should be expressed in terms of the porosity $\Phi(z, t)$ from (50), using appropriate boundary conditions; this expression of $u$ should be substituted into (51) and, of course, the permability $k$ should also be expressed in terms of $\Phi$ and some constants. It goes without saying that some of these constants (tortuosity, channel cross-section, etc.) are possibly also pressure-dependent (Volarovich, Marmorstein \& Mekler 1968), that is they can change during compaction! When all this is done, we get a partial differential equation for $\Phi(z, t)$ that in principle can be solved, at least numerically. It seems to us, however, that the solution of this equation and the derivation of an exact permeability-porosity dependence is a much more complex problem than the description of the final state of shale compaction studied in the present paper, and requires additional efforts. Just to show the potentialities of this approach for compaction problems, let us consider two simple cases when the consolidation equation can be readily solved for the equilibrium state (i.e. for $t \rightarrow \infty$ ). First, suppose that

$\Phi(z, t)=m_{\mathrm{vc}} \cdot u(z, t)+\phi_{1}$ 
where $\phi_{1}$ is a constant, and suppose further that the permeability $k$ is constant. Then $(50)$ is obviously satisfied while (49) becomes, in terms of $\Phi$ :

$\frac{\partial \Phi(z, t)}{\partial t}=\frac{k}{\gamma_{\mathrm{w}} m_{\mathrm{vc}}} \cdot \frac{\partial^{2} \Phi(z, t)}{\partial z^{2}}$.

In the final stage of compaction (for $t \rightarrow \infty$ ) the porosity of the shale does not change with time any more, that is the lhs of (53) tends to zero as $t \rightarrow \infty$ and we are left with

$\frac{\partial^{2} \Phi(z, t=\infty)}{\partial z^{2}}=0$.

The solution of (54) is

$\Phi(z, t=\infty)=A z+B$

where the constants $A$ and $B$ should be determined from the boundary conditions.

The linear porosity -depth dependence of (55), of course, does not reproduce Athy's law. It should be mentioned, however, that linear porosity-depth laws are generally accepted for the compaction of sands and sandstones (Maxwell 1964; Atwater \& Miller 1965), and occasionally have even been reported for shales (Sz. Kilényi 1968: Vikhirov 1975).

As the next example, suppose again that $\Phi$ and $u$ are connected by (52) and that the permeability is related to porosity according to the simple formula

$k=k_{0} \cdot \Phi^{\gamma}$

where $k_{0}$ and $\gamma$ are positive constants. Equation (51) becomes

$\frac{\partial \Phi(z, t)}{\partial t}=\frac{k_{0}}{m_{\mathrm{vc}} \gamma_{\mathrm{w}}} \frac{\partial}{\partial z}\left[\Phi^{\gamma}(z, t) \frac{\partial}{\partial z} \Phi(z, t)\right]$.

Observing, that

$\Phi^{\gamma} \frac{\partial}{\partial z} \Phi=\frac{1}{1+\gamma \partial z}\left(\Phi^{1+\gamma}\right)$

and again letting $t \rightarrow \infty$, the lhs of (57) tends to 0 and we obtain:

$\frac{\partial^{2}}{\partial z^{2}} \Phi^{1+\gamma}(z, t=\infty)-0$

that is

$\Phi(z, t=\infty)=(A z+B)^{1 / 1+\gamma}$

where $A$ and $B$ are appropriate constants. Since pressure is also linearly related to depth (Hedberg 1936), (59) has a similar form as Terzaghi's (1925) porosity--pressure relationship (cf. equation 4).

\section{Conclusions and outlook}

After a short problem discussion and historical outline of the ideas on shale compaction we presented what we believe to be the first mathematical-physical derivation of the hitherto only empirically established exponential porosity-depth dependence of compacted shales. 
Several analogies to the shale compaction law from different branches of physics have been offered. The main result of this paper is that the exponential compaction law expresses the maximum-entropy statistical equilibrium state of the pores in the rock, that is compaction is an irreversible process where clay particles tend towards a statistically defined final equilibrium state. Since it is a general law of nature that all closed systems strive towards maximum entropy, this tendency observed and proved here for shales should also be present in the evolutionary history of more complicated multicomponent geological media such as sand-shale interbeddings - let alone whole sedimentary basins. Of course for these more complicated problems, the entropy concept and the different constraints must be carefully redefined. Finally, due to the intimate connection between compaction, water expulsion and oil/gas migration (Athy 1930b; Magara 1976), studies of these type could have a bearing on the more practical problems of hydrocarbon migration.

\section{Acknowledgments}

I would like to express my sincere thanks to the two unknown referees who kindly pointed out the deficiencies in the original derivation of equation (25), and called my attention to the necessity of reconciling the statistical approach with the consolidation theories of soil mechanics.

\section{References}

Anstey, N. A., 1977. Seismic Interpretation: the Physical Aspects, International Human Resources Development Corporation, Boston.

Athy, L. F. 1930a. Density, porosity, and compaction of sedimentary rocks, Bull. Am. Ass. Petrol. Geol., $14,1-24$.

Athy, L. F., 1930b. Compaction and oil migration, Bull. Am. Ass. Petrol. Geol., 14, 25-35.

Atwater, G. I. \& Miller, E. E., 1965. The effect of decrease in porosity with depth on future development of oil and gas reserves in South Louisiana, Prog. Ann. mtg, geol. Soc. Am., New Orleans, p. 48.

Avchyan, G. M. \& Ozerskaya, M. L., 1968. Compaction of sedimentary rocks as a function of depth, Izv. Akad. Nauk. SSR Ser. Geol, no. 2 (in Russian).

Baldwin, 1971. Ways of deciphering compacted sediments, J. sedim. Petrol., 41, 293.

Ceccherini, P. T. \& Finetti, I., 1968. Density computation problem of compactable rocks for gravity reduction, Boll. Geofis. teor. appl., 10, 281-289.

Cordell, L., 1973. Gravity analysis using an exponential density-depth function - San Jacinto Graben, California, Geophysics, 38, 684-690.

Dabrowski, A., 1976. Mean densities of Pre-Devonian sedimentary rocks in Poland and their depth dependence, Pure appl. Geophys., 114, 251-262.

Dickinson, G., 1953. Geological aspects of abnormal reservoir pressures in Gulf Coast Louisiana, Bull. Am. Ass. Petrol. Geol., 37, 410-432.

Floyd, E. R., 1981. Thermodynamic corrections to the velocity of propagation in a bubbly medium, J. acoust. Soc. Am., 70, $1748-1751$.

Fritz, J., 1967. Information theory and thermodynamics of gas systems, Proc. Coll. Information Theory, pp. 167-175, September 19-24, Szeged, Hungary.

Gladkikh, Yu. P. \& Urazaev, B. M., 1972. Correlations between the physical properties of the rocks and the depth of their burial, Izv. Akad. Nauk. Kaz. SSR. Ser. Geol., 6, 15-31 (in Russian).

Grant, F. S. \& West, G. F., 1965. Interpretation Theory in Applied Geophysics, McGraw-Hill, New York.

Hamilton, E. L., 1970. Sound velocity and related properties of marine sediments, North Pacific, J. geophys. Res., 75, 4423-4446.

Hamilton, E. L., 1980. Geoacoustic modeling of the sea floor, J. acoust. Soc. Am., 68, 1313-1340.

Hedberg, H. D., 1926. The effect of gravitational compaction on the structure of sedimentary rocks. Bull. Am. Ass. Petrol. Geol., 10, 1035-1072.

Hedberg, H. D., 1927. The effect of gravitational compaction on the structure of sedimentary rocks; a reply to a discussion by W. W. Rubey, Bull. Am. Ass. Petrol. Geol., 11, 875-886.

Hedberg, H. D., 1936. Gravitational compaction of clays and shales, Am. J. Sci., 31, 241-287. 
Howell, L. G., Heintz, K. C. \& Barry, A., 1966. The development and use of a high-precision downhole gravity meter, Geophysics, 18, 298-309.

Kapolyi, L., 1980. A system theoretical approach to the utilization processes of mineral resources, Inaugural Speech at the Hungarian Academy of Sciences, February 24, Trans. Hung. Acad. Sci, class $X, 13,259-300$ (in Hungarian).

Károlyházi, F., Marx, G. \& Nagy, K., 1965. Statistical Mechanics, Technical Publishing House, Budapest (in Hungarian).

Kneschke, A., 1960. Differentialgleichungen und Randwertprobleme, I, VEB Verlag Technik, Berlin.

Kopf, M., 1967. Der Dichteansatz für das Norddeutsch-Polnische Becken unter besonderer Berücksichtigung des vertikalen Dichtegradienten, Geologie, 16, no. 2.

Landau, L. D. \& Lifshitz, E. M., 1981. Statistical Physics, I, Nauka, Moscow (in Russian).

Litwiniszyn, J., 1974. Stochastic Methods in the Mechanics of Granular Bodies. International Centre for Mechanical Sciencies, Courses and Lectures no. 93, Udine, Springer-Verlag, Wien.

Macke, W., 1962. Thermodynamik und Statistik. Akad. Verlagsgesellschaft. Geest \& Portig K.G., Leipzig.

Magara, K., 1976. Water expulsion from clastic sediments during compaction - directions and volumes, Bull. Am. Ass. Petrol. Geol., 60, $543-553$.

Matthews, J. E., 1980. Heuristic physical property model for marine sediments, J. acoust. Soc. Am., 68, $1361-1370$.

Maxwell, J. C. \& Verrall, P., 1954. Low porosity may limit oil in deep sands, Whl Oil, April, 106-113.

Maxwell, J. C., 1964. Influence of depth, temperature and geological age on porosity of quartzose sandstone, Bull. Am. Ass. Petrol. Geol., 48,697-709.

McCulloch, T. H., 1965. A confirmation by gravity measurements of an underground-density profile based on core densities, Geophysics, 29, 1108-1132.

Medwin, H., 1970. In situ acoustic measurements of bubble populations in coastal ocean water, $J$. geophys. Res., 75, 599-611.

Militzer, H. \& Lindner, H., 1976. Grundlagen der Angewandten Gravimetrie, Bergakademie Freiberg, Sektion Geowissenschaften.

Nafe, J. E. \& Drake, C. L., 1957. Variation with depth in shallow and deep water marine sediments of porosity, density and the velocities of compressional and shear waves, Geophysics, 22, 523-552.

Nagumo, S., 1965. Compaction of sedimentary rock - a consideration by the theory of porous media, Bull. Earthq. Res. Inst., Tokyo, 43, $339-348$.

Ozerskaya, M. L., 1965. The effects of structural factors on the density and elastic properties of sedimentary rocks, Izv. Akad. Nauk. SSSR Fiz. Zemli, no. 1, 103-108 (in Russian).

Ozerskaya, M. L. \& Semonova, S. G., 1973. The effect of the state of sedimentary rocks on the depthdependence of their physical properties, Prikl. geofiz., 70, 186-191 (in Russian).

Reed, M. \& Simon, B. 1972. Methods of Modern Mathematical Physics. I. Functional Analysis, Academic Press, New York.

Rische, H., 1957. Dichtebestimmungen im Gesteinsverband durch Gravimeter- und Drehwaagemessungen unter Tage, Freiburger ForschHft., C35, Berlin.

Rubey, W. W., 1927a. The effect of gravitational compaction on the structure of sedimentary rocks: a discussion, Bull. Am. Ass. Petrol. Geol., 11, 621-633.

Rubey, W. W., 1927b. The effect of gravitational compaction on the structure of sedimentary rocks, Bull. Am. Ass. Petrol. Geol., 11, 1333-1336.

Scheidegger, A. E., 1963. Hydrodynamics of porous media, in Handbuch der Physik, VIII/2, 626-628.

Schopper, J. R., 1966. A theoretical investigation on the formation factor/permeability/porosity relationship using a network model, Geophys. Prospect., 15, 301-341.

Sommerfeld, A., 1962. Thermodynamik und Statistik. Akad. Verlagsgesellsch. Geest \& Portig K.G., Leipzig.

Sz. Kilenyi, E, 1968. Geological-geophysical conclusions on the Great Hungarian Plain through a statistical analysis of density data, Geophys. Trans., 17, $41-49$ (in Hungarian).

Terzaghi, K., 1923. Die Berechnung der Durchlässigkeitsziffer des Tones aus dem Verlauf der hydrodynamischen Spannungserscheinungen, Sher. Akad. Wiss. Wien, Abt. II.a., 132.

Terzaghi, Ch., 1925. Principles of soil mechanics. IV. Settlement and consolidation of clay, Engng News Rec.. 95. 874-878.

Terzaghi, K., 1965. Theoretical Soil Mechanics, Chapman \& Hall, London.

Terzaghi, K. \& Fröhlich, O. K., 1936. Theorie der Setzung von Tonschichten, F. Deuticke, Vienna.

Tipper, J. C., 1983. Rates of sedimentation, and stratigraphical completeness, Nature, 302, 696-698.

Vikhirov, B. V., 1965. On the relation between density and total porosity of sedimentary rocks, Prikl. geofiz., 43, $92-113$ (in Russian). 
Vikhirov, B. V., 1975. Mathematical models applied for the density studies of rocks, Prikl. geofiz., 77, $155-166$ (in Russian).

Vikhirov, B. V. \& Taraskova, E. O., 1975. A method for the study of the relationship between density and porosity of rocks, Prikl. geofiz., 79, 171-180 (in Russian).

Volarovich, M. M., Kurskeyev, A. K., Levykin, A. I., Tomashevskaya, I. S., Tuzova, I. L. \& Urazaev, B. M., 1970. High-pressure studies on the density of rocks from Central Kazakhstan, Izv. Akad. Nauk. SSR Fizika Zemli, 1, 46-51 (in Russian).

Volarovich, M. P., Marmorstein, L. M. \& Mekler, Yu. B., 1968. Variation in the structure of the pore space in sandstones under pressure, Izv. Earth Phys., 6, 15-19.

Walker, J., 1982. Why do particles of sand and mud stick together when they are wet? Sci. Am., January, $144-\cdots 149$.

Watts, A. B., 1981. The U.S. Atlantic continental margin: subsidence history, crustal structure and thermal evolution, in Geology of Passive Continental Margins: History, Structure and Sedimento. logic Record, Am. Ass. Petrol. Geol. Educ. Course Note Series, no. 19, 75 pp.

Weyl, W. A. \& Ormsby, W. C. 1960. Atomistic approach to the rheology of sand-water and of clay-water mixtures, in Rheology: Theory and Applications, vol. 3, ed. Eirich, F. R., Academic Press, New York.

Wyllie, M. R. J. \& Rose, W. D., 1950. Some theoretical considerations related to the quantitative evaluation of the physical characteristics of reservoir rock from electrical data, Trans. Am. Inst. Min. Engrs, 189, 105.

Zseller, P., 1980. Depth-dependence of the interval velocity in normally compacted sediments, 25 th int. Geophysical Symp., I, 119-125, Székesfehérvár, September 16-20. 